\title{
UNA REFLEXIÓN SOBRE LA ETNOMATEMÁTICA DEL ALBAÑIL Y LAS MATEMÁTICAS DE LA ESCUELA
}

\section{ARTÍ́CULO ORIGINAL}

SCHWANTES, Vilson ${ }^{1}$

XAVIER, Márcio Pizzete ${ }^{2}$

SCHWANTES, Eloísa Bernardete Finkler ${ }^{3}$

SCHWANTES, Daniel ${ }^{4}$

${ }^{1}$ Master en Ciencias de la Educación - Matemáticas, UNIJUI - RS. Licenciatura y Especialización en Ciencias y Matemáticas. Prof. Asistente del CCA - Centro de Ciencias Agrícolas, Campus del Mariscal Cândido Rondon, UNIOESTE, PR - Brasil.

2 Máster en Desarrollo Rural Sostenible-UNIOESTE, Especialista en Matemáticas, Física-UNIPAR, Gestión de Personas y Educación Especial con énfasis en Discapacidad Múltiple-UNIASSELVI, Graduado en Matemáticas con énfasis en FísicaUNIPAR.

${ }^{3}$ Especialización en Enseñanza de Ciencias Exactas - Matemáticas, Física y Química. UNIOESTE - Universidad Estatal de Paraná Occidental. Graduación: Ciencias y Matemáticas. UNIJUI, RS. Profesor de la Escuela Estatal de Paraná.

${ }^{4}$ Profesor de Protección Fitosanitaria y Salud Humana en la Pontificia Universidad Católica de Chile, Departamento de Ciencias Vegetales, ocupa un puesto interdisciplinario compartido entre la Facultad de Agronomía e Ingeniería Forestal (FAIF), la Facultad de Medicina y la Facultad de Química. Profesor investigador asociado al Grupo de Estudio sobre Suelos y Medio Ambiente (GESOMA UNIOESTE). Máster en Agronomía por UNIOESTE, Doctorado en Agronomía por UNIOESTE (2013-2016) - Periodo Sandwich (Beca CAPES) de la Universidad de Lisboa, en el Instituto Superior de Agronomía (ULisboa). 
JUNIOR, Affonso Celso Gonçalves ${ }^{5}$

KRACKE, Elisa ${ }^{6}$

JUNIOR, Élio Conradi ${ }^{7}$

SCHWANTES, Vilson. Et al. Una reflexión sobre la etnomatemática del albañil y las matemáticas de la escuela. Revista Científica Multidisciplinar Núcleo do Conhecimento. año 04, Ed. 07, Vol. 14, págs. 87-106. Julio de 2019. ISSN: 2448-0959

\section{RESUMEN}

Este artículo reflexiona sobre Etnomathematics, analiza entrevistas con un grupo de albañiles y vendedores de material de construcción de los municipios de Mercedes y Marechal Cándido Rondon, PR. Busca conocer los conocimientos matemáticos utilizados por estos trabajadores en el ejercicio de sus profesiones y su posible relación con la enseñanza de las matemáticas escolares. En los informes de los participantes parece que estos conocimientos están íntimamente ligados al entorno

5 Productividad de investigación Nivel $1 \mathrm{C}$ por CNPq en el área de Ciencias Ambientales con tres Postdocs, UEM-PR (Brasil), Universidad de Santiago de Compostela (España), UFG-GO (Brasil). Actualmente es profesor asociado en UNIOESTE-PR y actúa como profesor e investigador en el Centro de Ciencias Agrarias, enseñando química. Profesor del Programa de Maestría en Ciencias Agrícolas de la UEM. Actualmente es consultor ad hoc para CNPq, CAPES y Fundação Araucária. Actúa como consultor ambiental voluntario con MP-SP y CONAMA-DF.

${ }^{6}$ Licenciado en Agronomía - UNIOESTE - Universidad Estatal de Paraná Occidental Licenciado en Administración - Unip - Universidade Paulista.

7 Estudiante de maestría en Agronomía (Producción de Plantas) de la Universidad Estatal de Paraná Occidental (UNIOESTE). Ingeniero Agrónomo egresado de UNIOESTE (2014-2018), actúa como investigador asociado al Grupo de Estudio de Suelos y Medio Ambiente (GESOMA - UNIOESTE). 
sociocultural, denotando una concepción de que la escuela es un lugar privilegiado donde el conocimiento matemático se aprende de una manera organizada y sisticada. necesaria según la vida profesional. Es evidente que hay retos que debe superar la escuela para su consolidación efectiva, de modo que la práctica pedagógica de la educación escolar tenga conexiones con el mundo sociocultural del estudiante.

Palabras clave: perspectiva de etnomatemática, educación matemática, conocimientos matemáticos, rutina en el aula.

\section{INTRODUCCIÓN}

La enseñanza de las matemáticas en las escuelas brasileñas, en las últimas décadas, ha sido predominantemente tradicional, sin cambios pedagógicos importantes, excepto por algunas excepciones. Dentro de esta perspectiva y realidad se percibe que poco se utiliza de los Sabres anteriores de los estudiantes, y menos aún se hacen relaciones de los conceptos matemáticos que se enseñan con la vida de los alumnos fuera del entorno escolar. En este escenario todavía es común escuchar en el entorno escolar expresiones tales como: "Maestro donde usaré esto en mi vida?".

Según Santos (2016):

La falta de contextualización en la enseñanza de las matemáticas puede conducir al desánimo de la disciplina, resucitando los métodos tradicionales que conceptualizan las matemáticas como una ciencia que preparó todas las cosas, como si fuera un conocimiento listo y Sobre. ( $p$. 5)

En muchas escuelas brasileñas, todavía se escucha que la enseñanza de las matemáticas es distante y descontextualizada de la realidad experimentada por los estudiantes y los ciudadanos. Para Demo (1996), la construcción del conocimiento parte del conocimiento incorporado por cada individuo en su experiencia sociocultural. Para el autor "Todos hablan, se comunican, utilizan un vocabulario básico, gestionan 
conceptos dentro del sentido común, tienen referencias de la realidad en la que se insertan" (pág. 32).

Aunque todavía estamos experimentando este tipo de situaciones en nuestras escuelas, hay un consenso de que los estudiantes que ingresan a la escuela traen a su equipaje algún conocimiento, incluso si es sólo dentro del sentido común. Esto incluye el conocimiento matemático generado en la familia y en los sectores de la sociedad, que es incorporado por los niños y es esencial que la escuela lo conozca y lo considere, basándose en estos conocimientos, el conocimiento del sistema. Para Pompeyo y Monteiro (2001), "Los estudiantes aprenden en la vida cotidiana, en su relación con el mundo" (pág. 62).

Para Lopes (2014),

"Las ideas y situaciones de carácter matemático están presentes en las cosas cotidianas, en las actividades profesionales, en las prácticas de diferentes culturas, en situaciones de conteo, medición y cálculo, que son fácilmente reconocidas como matemáticas." (p.5)

Una enseñanza con la producción de significados sobre el contexto es tan fundamental como la relación con el mundo real, el mundo abstracto y simbólico. Partiendo de la experiencia cultural de cada alumno, el significado que aporta de su experiencia social y cultural, uno puede avanzar y entender otras culturas, individuos y sociedad. Compartimos con D'ambrosius (1993), cuando postuló que es fundamental entender que cuando comienza la educación formal, el niño ya tiene una etnomatemática que le permite enfrentar las interpretaciones de las matemáticas sistemáticas de la escuela.

Esta perspectiva, según Marqués (2001), representa y significa:

Construcción de nuevos conocimientos, basados en conocimientos previos; De hecho, una reconstrucción de ellos, en el sentido de desmontaje y recuperación de una nueva manera. El conocimiento de 
cada interlocutor-confidante, lectores, autores convocados con sus obras, temas de prácticas sociales a los que escuché, entrevisté, azul-y mi conocimiento se fusiona y transforma, reformula. De una manera muy especial, mis conocimientos previos ahora configuran otros. Eso es lo que llamamos aprender. ¿Por qué no investigar? (P. 26).

Compartimos con Fiorentini (1995) destacando que en el enfoque etnomathematics, el proceso de enseñanza-aprendizaje tiene como punto de partida el énfasis dado a los problemas que de alguna manera tienen sentido para los niños. En este sentido, la relación alumno-profesor se produce en el contexto de la intersubjetividad, donde a través del diálogo, la interlocución entre asignaturas, el intercambio de experiencias, ambos (re) construyen conocimientos mediante La Problematización (mathemtización) sobre el sentido común y el conocimiento Systate, construyendo una nueva mirada al contexto.

Aunque está claro que la escuela necesita trabajar en el conocimiento matemático sistemático, creemos que una apreciación de los conocimientos matemáticos resultantes de las relaciones culturales de los estudiantes puede hacer que el crecimiento sea positivo y psicológico Los ingresos de los niños, en contraposición a una iniciación a través de técnicas y reglas para llegar a respuestas correctas. Esta posibilidad se puede vislumbrar y concretar con la adopción por los educadores matemáticos de una postura etno en la enseñanza, porque según Pompeyo y Monteiro (2001), "La etnomatemática busca una transformación de las relaciones actuales del hombre con el mundo" (p. 148 ).

Para actuar en el mundo de hoy, es necesaria una nueva postura, un nuevo paradigma, una revisión teórica que tenga como objetivo central la Organización/reformulación/comprensión de la base teórica y práctica que impregna la acción pedagógica de los Educadores (SILVA, 2012, p. 1744 ). 
Para D'ambrósio (2001), la Etnomatemática puede ayudar en la Constitución del investigador profesor de su práctica, despertándolo de manera continua para ser profesor, de acuerdo con las necesidades educativas actuales, transformando el aula en un ambiente intercambio de conocimientos, rescatando el conocimiento históricosocialmente construido de la humanidad.

Los proyectos pedagógicos escolares carecen de adecuación al nuevo paradigma educativo, centrado en la intersubjetividad y la interlocución de los actores. Un enfoque matemático en esta perspectiva transforma el aula en un ambiente de intercambio de conocimiento al valorar las diferencias individuales, porque la redención de la curiosidad recupera el conocimiento histórico-socialmente construido por el hombre, a lo largo de la Historia de la humanidad.

Reflexiones y discusiones sobre la enseñanza de esta disciplina, también consiste en verificar las expectativas y necesidades sociales de las matemáticas enseñadas en la escuela, según las palabras de Demo (1996, p. 10), es "salir de la postura reproductiva golpeada, marcada Especialmente para que la clase repetida repita. "

Las nuevas tendencias en la educación y la educación matemática enfatizan que el arte de aprender a enseñar implica no sólo saber qué enseñar y saber acerca de los métodos de enseñanza, sino, sobre todo, implica un conocimiento sobre el aprendizaje. Nos referimos al aprendizaje, tanto del maestro, sobre el proceso en sí y los resultados de su aprendizaje, como de un conocimiento del maestro en relación con el aprendizaje de sus alumnos. (SILVA, 2012, p.197).

Así, enseñar matemáticas en el aula, teniendo en cuenta los conocimientos previos y la diversidad cultural de los estudiantes puede ser una estrategia importante para una enseñanza más significativa y menos tradicional de las matemáticas escolares, generando más interés y Participación de los estudiantes en la construcción de conocimientos matemáticos. 
Creemos que una postura didáctica-pedagógica que parte del conocimiento que el estudiante ya tiene de su experiencia diaria, proporciona a los educadores una (re) construcción del conocimiento matemático systate, tanto en la percepción de la existencia de varios Matemáticas en medio de nosotros, así como la búsqueda de una enseñanza que contemple las expectativas sociales.

\section{JUSTIING THE PRESENCE OF MATHEMATICS IN THE DAILY ACTIVITY OF THE MASON}

Cuando actuamos en diferentes momentos, las sesiones de estudio con los albañiles, buscamos investigar de forma dialogante con cada profesional, sus opiniones sobre cuestiones matemáticas de carácter general que impregnan su peaje diario.

Cuestionados sobre el uso de las matemáticas en su trabajo, los albañiles fueron unánimes al afirmar que es fundamental en el ejercicio de su profesión. Según Oscar, "principalmente al comienzo de una obra, el alambre de plomada. Con el alambre de plomada hacemos una matemática no tan complicada como la de la escuela y sin fórmulas, complementa al Masón. Lo que más usamos es razonar, enfatizar.

Sérgio comenta que utiliza las matemáticas "para medir y calcular la cantidad de azulejos, ladrillos, a veces metros cuadrados. Son cálculos de tiemp[multiplicação]os, má[adição]s y división. " Alberto también dice usando matemáticas "desde la fundación de la construcción, para sacar la plaza, a través del teorema de Pitágoras. En el techo para hacer caer el porcentaje del agua. También uso las matemáticas en el área mide "fines.

Cuando argumentamos que usaban una matemática práctica, Oscar pronto dijo que en el ejercicio de una profesión una matemática "más práctica y simple" no se utiliza en las fórmulas que se enseñan en la escuela. Siempre tratamos de simplificar los procedimientos. En una construcción definiremos el tipo de cálculo que utilizará. No perdemos mucho tiempo en cálculos complicados, todo se hace de una manera más simplificada, argumenta. 
Instigado a aclarar mejor las matemáticas que utiliza y le permite hacer cálculos de forma simplificada, Oscar informó que es "un razonamiento que es prácticamente el día a día del constructor e implica especialmente medidas. Nos preocupamos por ellos todo el día y, usted necesita estar siempre atento. "

Según Breda, Lima y Guimarés (2011, p. 15):

Empecé a ver la propuesta de Etnomathematics como una posibilidad de diferenciar el trabajo que el profesor desarrolla en las escuelas, es decir, la práctica conteudista y sin sentido puede ser sustituta por un maestro orientado por una nueva mirada, que fomente La apreciación del contexto sociocultural de la educación, sus procesos de pensamiento y sus formas de entender, explicar y ejercer su práctica en la sociedad contemporánea u[...]na invitación a revisitar sus prácticas pedagógicas y sus efectos o incluso mirar su papel, Como profesor que se ocupa de diferentes perspectivas de la asignatura en el contexto escolar.

Para la investigación, consideramos interesante preguntar dónde y cómo aprendieron estas matemáticas que utilizan los algoritmos de las operaciones, pero trae subyacente un tipo de razonamiento que permite la resolución de las situaciones problemáticas que enfrentan diariamente. Sérgio dice que aprendió "en el día a día, con la gente que aprendí a trabajar como albañil. Empecé como ayudante y aprendí hasta que llegué a este punto de ser autónomo".

El Mason Oscar, en su informe, dice que aprendió "en el curso primario lo básico, pero un gran porcentaje de lo que usamos, aprende de la obra en sí. A medida que aparezcan los problemas, resolverá saque sacon se adiario de la construcción cotidiana. " Según Pompeu y Monteiro (2001), esta postura refleja la adopción de la concepción empírica, utilizando las matemáticas para resolver las situaciones problemáticas más inmediatas que impregnan la vida cotidiana de la actividad del albañil. Lo fundamental, en esta perspectiva según los autores, es resolver los problemas prácticos a los que se enfrenta el peaje diario de la profesión. 
Cuando se le pidió que dijera cómo aprendió los procedimientos necesarios para ejercer esta profesión, Oscar informó que "al principio trabajaba como auxiliar y estaba observando cómo lo hacían otras personas. Creo que la persona tiene que tener interés y en el tiempo aprende y perfecciona lo que hace, encontrando otras maneras de hacer los cálculos. No puedes estar quieto, tienes que ser curioso dentro de lo que haces, tratando de prestar la máxima atención a perfeccionar y ser cada vez más objetivo en el trabajo que realizas. Según D'ambrósio (2001) Un comportamiento que refleja "la aventura de la especie humana en la búsqueda del conocimiento y en la adopción de procedimientos" (p. 17).

"Siempre fui muy dedicado a mi padre, que era albañil, era muy noser, así que aprendí muchas cosas de él. Muchas cosas también aprendí en el día a día, pero sin las matemáticas de la escuela sería difícil ejercer mi profesión, porque ayuda mucho", concluye Alberto. Según Marques (2000) "Los procesos de aprendizaje se insertan inevitablemente en comunidades comunicativas y públicas en las que los hombres aprenden unos de otros y unos con otros" (pág. 29).

Las matemáticas deben interpretarse como un producto sociocultural-natural de un pueblo, según Rosa Neto (2002, p. 7 y 19).

Las matemáticas fueron creadas y ha sido desarrollada por el hombre de acuerdo a sus necesidades. (...) La cultura es una forma de adaptación porque es una forma de actuar sobre el medio ambiente que se construyó junto con ella.

Para Rosa Neto (2002), existe una relación significativa entre matemáticas y cultura, tanto como resultado de nuestra adaptación según nuestras necesidades de supervivencia a través de los tiempos, representando tanto a Científica.

Teniendo en cuenta que los masones que participan en la investigación consideran que las matemáticas de las que utilizan, en el ejercicio de la profesión, satisfacen sus necesidades profesionales $y$, debido a que han sido aprendidas en su formación básica, en el peaje diario con otros Los albañiles más experimentados, consideramos 
pertinente preguntar si creían que las matemáticas de la escuela podrían mejorar las matemáticas que usan a diario.

En los discursos, demostraron creer que la escuela es un lugar donde de manera organizada e intencional se procesa la sistematización del conocimiento incautado dentro o fuera de sus fronteras territoriales. Oscar declaró: "Creo que las matemáticas de la escuela mejorarían mis cálculos, y podría hacer las cosas más fácilmente al salir de la escuela todo el tiempo. Las matemáticas que se enseñan en la escuela son válidas y sirven como punto de partida, ayudando en lo que hacemos. Aunque normalmente siempre elegimos hacer lo que aprendiste en la vida cotidiana" En el discurso del Masón, según Giardinetto (1999) aparece que "el acceso al conocimiento matemático sistemático ha sido indispensable para la transformación de la vida cotidiana" (pág. 7).

A través de estas enunciaciones, es evidente la presencia de un entendimiento que se apoya en las Reflexiones de Giardinetto (1999), destacando que, de hecho, "el conocimiento escolar supera el modo de pensamiento presente en la vida cotidiana a partir de elementos, gérmenes Presente en la vida cotidiana y que se incorporan por el conocimiento escolar "(pág. 50). Según el autor, "la formación de todo hombre va mucho más allá de lo que determinaron las actividades práctica-utilitarias de la vida cotidiana" (pág. 78).

Para Sergio, "sin las matemáticas de la escuela, incluso las cuatro operaciones básicas se volverían difíciles". Esto demuestra, según Giardinetto (1999) que "el conocimiento mismo que cada individuo prepara para su vida diaria no tiene en cuenta responder a las necesidades de su propia vida diaria" (pág. 7).

Sin embargo, cuando se le preguntó sobre cómo ve las matemáticas que se enseñan actualmente en la escuela, Sergio postuló que ayuda a las personas y es necesario. Enfatizó, sin embargo, que tiene dificultades para opinar con mayor profundidad: "No conozco las matemáticas de la escuela. Sólo lo hice hasta el cuarto grado y mis hijos son todavía pequeños ", concluye. 
Según Oscar, las matemáticas de la escuela de hoy en día son "muy complejas y muy complicadas. Hay muchas fórmulas y en el día a día no se pueden aplicar, por lo que poco guardar las fórmulas en la memoria para hacer los cálculos. Sería importante enseñar matemáticas más convenientemente, más en lo que usará en su día a día en cualquier sector. Para mi trabajo de construcción, creo que estas fórmulas escolares tienen poca relación con lo que voy a necesitar todos los días"

Reflexionando sobre la importancia de relacionar el contenido matemático con una situación real, Alberto comenta: "Es cierto, como en el caso del teorema de Pitágoras, hace años para eliminar la plaza de la casa, utilizamos la propia plaza y se estaba alineando hasta Consigue el material de archivo correcto. Ahora, recientemente surgió para nosotros los $3 \mathrm{~m} ; 4 \mathrm{~m}$ y $5 \mathrm{~m}$ que también sale de la casa en la plaza. Aprendí en la escuela que este es el teorema de Pitágoras, quiero decir, haciendo la medida de $3 \mathrm{~m}$; $4 \mathrm{~m}$, la diagonal con $5 \mathrm{~m}$ pone todo en el cuadrado ".

Cuando se está instigando en las matemáticas que se enseñan actualmente en la escuela Alberto dice que "ella es muy teórica, debe aportar más la parte práctica. Trabajar la práctica, no sólo la teoría, esto sería muy importante para el niño, ayuda en el desarrollo intelectual. La niña que tiene lo teórico y lo práctico que entrará en el campo profesional más inteligente", concluye el albañil.

Y su discurso, Oscar todavía informa que en el ejercicio de su profesión "muchas veces podemos resolver ciertos cálculos a través de otras fórmulas más simples que las enseñadas por la escuela". Ciertamente para estos casos, debemos comportarnos como sugieren Lins y Giménez (1997), es decir, "de lo que es más familiar" (p. 10). Con esta postura didáctica, según los autores, lo que se aprendería en la escuela, no serviría "sólo para la escuela" (pág. 17), porque tendrían una relación directa con los sectores de la actividad diaria.

Al ser instigado a hablar de cómo les gustaría que les enseñaran las matemáticas de la escuela, Alberto dice fácilmente: "Con ejemplos prácticos, buscando aplicaciones en diversas profesiones: comercio, albañiles, carpinteros, entre otros. Problemas 
prácticos para no enseñar matemáticas sólo a través de la resolución del ejercicio. Estos problemas matemáticos pueden hacer que el estudiante asimile la teoría y entender todo mejor, de lo contrario no tiene sentido. Aprendí el teorema de Pitágoras en la escuela, pero el maestro no mostró que sirviera en el día a día, completa el albañil.

Las enunciaciones de los Albañiles muestran, según la perspectiva de Lins (1994), en sus estudios sobre el modelo teórico de campos semánticos "que el mismo texto, hablado con diferentes justificaciones, constituye un conocimiento diferente ${ }^{[8] "}$ (p. 29).

Para finalizar la reflexión que hemos propuesto, les dejamos a gusto para decir algo más sobre las matemáticas que se enseñan en la escuela y las matemáticas que utilizan en el ejercicio de su profesión. Oscar volvió a priorizar en su discurso la importancia de las matemáticas escolares: "Los fundamentos que aprendí en la escuela y uso hoy en mi profesión. Las matemáticas de los libros que usas hoy en día son más avanzadas y particularmente no uso mucho en mi trabajo, pero puede ser utilizado en otras áreas y en otros sectores.

Las matemáticas que aprendí en la escuela, y mis padres han aprendido a usar mucha memoria. Hoy usamos las fórmulas y la calculadora. Incluso uso ninguna calculadora, prefiero trabajar con memoria, aunque la calculadora es mucho más práctica. En mi trabajo, en muchas situaciones necesito definir las cosas con la capacidad de la memoria, capturar cosas, terminar".

Según Alberto las matemáticas de la escuela "ayuda mucho. Fue muy bueno para mí estudiar. La experiencia que tuve con mi padre fue muy importante, pero sin escuela, no lo hace. Resolver todo en el grito no funciona. Creo que las matemáticas de la escuela tienen una importancia muy grande en mi profesión. Las matemáticas de la escuela me ayudaron a evitar muchos errores que había cometido antes. Todo el trabajo realizado en la construcción se realiza con algún tipo de matemáticas. "

Según D'ambrósio (2001), el testimonio del Mason muestra que, "Al menos te confunde con el educador matemático que no se da cuenta de que hay mucho más 
en su misión como educador que la enseñanza para hacer contins o resolver ecuaciones y problemas absolutamente Artificial, incluso si a menudo tiene la apariencia de estar refiriéndose a hechos reales "(p. 46).

En cuanto a la importancia dispensada a las matemáticas impartidas por la escuela, Oscar fue enfático, afirmando que "no podemos descartar las matemáticas de la escuela porque enseña los principios de los cálculos y sin ellos todo se complica. Resulta que las matemáticas de la escuela se complican debido a muchas fórmulas y esto, para el albañil, trae un cierto descontento entre los propios estudiantes.

Para Rolim,

Pensar en el contexto histórico-cultural en el proceso de enseñanza del aprendizaje de las matemáticas es considerar que "hacer" implica más que reglas y técnicas; reconocer que como ciencia, las matemáticas son en sí mismas un edificio de la humanidad (ROLIM, 2010, p. 43).

Con el desinterés la estudiante escapa de las matemáticas, está aterrorizada y esto debe tener una relación con la forma en que se le enseña. No creo que deba hablar mucho de este tema porque no fui demasiado a la escuela. Lo que dije es por lo que he seguido en la vida escolar de mis hijos, tratando de ayudarlos en algunas ocasiones, y puedo decir que no he sido capaz de resolver la mayoría de los problemas y problemas porque es difícil entender las fórmulas que se utilizan en la escuela hoy en día"

La afirmación inicial del albañil da la idea, según Giardinetto (1999) de que"es necesario entender que el conocimiento en la vida cotidiana es un conocimiento fragmentado que se manifiesta de acuerdo con una lógica conceptual propia de las demandas de toda la vida cotidiana. Esta es una lógica conceptual que es adecuada a los objetivos práctico-utilitarios y responde eficazmente a las necesidades de la vida cotidiana"(p. 6). 
Ante el discurso de Oscar, pregúntale si pensaba que a menudo la enseñanza de las matemáticas estaba lejos de ser una situación real, a lo que aceptó decir: "Exactamente, si miro las matemáticas que uso en mi trabajo y la que está en los libros , 99\% no uso en el día a día.

En este sentido creo que es necesario reflexionar sobre este tema y comprobar en el futuro cómo hacer las matemáticas modernas más aplicadas" Según Giardinetto (1999), se trata de entender la "actividad escolar como mediador de esta relación con el fin de rescatar el papel indispensable de la escuela para la formación del individuo, superando las interpretaciones superficiales e inmediatas que Sobrevaloran los conocimientos diarios en detrimento de la apropiación de los conocimientos escolares"(p. 12).

Según Marques (2000) "La educación en sus diversos casos, especialmente en la escuela, tiende a estandarizar el aprendizaje con miras a la producción del individuo modelado por la cultura actual". En vista de esta observación del autor, tratamos de mostrar que el conocimiento matemático existe y es utilizado por la gente de la comunidad, pero no de una manera única y estandarizada. Dentro de este escenario intersubjetivo, creemos que sólo un enfoque etno proporcionará a la enseñanza de las matemáticas escolares el debate discursiva sobre el conocimiento en circulación en las diversas actividades humanas, reconstruyendo ambas actividades (pág. 34).

Las matemáticas pueden, sin embargo, ocupar conocimientos y entendimientos que no encajan en las estructuras institucionalizadas por los planes de estudio y los programas de investigación. En este sentido, sería posible destacar las matemáticas presentes en el día a día de muchas profesiones. Es una parte integral de la tecnología, el diseño y la toma de decisiones, está en tablas, diagramas y gráficos. Simplemente navegue por un periódico para encontrar una gran cantidad de matemáticas (SKOVSMOSE, 2014, p. 13-14). 
La mediación docente, de acuerdo con la perspectiva de Vygotsky (2001) no monopoliza el discurso del aula, sin embargo, lo transforma en una comunidad argumentativa del aprendizaje, donde el papel de ambos es relevante en el proceso de (re) Constitución de los aprendizajes. En esta perspectiva, la participación de los alumnos en el aula y la participación de los sectores que componen la comunidad escolar se ampliarían, creando constantemente un clima de debates cognitivos entre ellos, que reconstruye y renueva el aprendizaje de ambos.

En las visitas que hicimos a tres tiendas que reenvían material de construcción, nos llamó la atención, tanto el buen servicio de los empleados, como a las manifestaciones de preocupación de los gerentes, en cuanto a la escasez de cursos de formación para los albañiles en Construcción en modo general y revestimiento cerámico en particular.

En una de las tiendas visitadas, cuando el tema central de la investigación llegó a los oídos de los líderes de la empresa, nos presentaron por primera vez al ingeniero civil y a él al gerente propietario. En el diálogo, escuchamos de ellos muchas ansiedades e inquietudes. Todas las preocupaciones se dirigieron al tratamiento descontextualizado dado a las matemáticas escolares, así como a la falta de su dominio por parte de los albañiles en general, en el ejercicio de sus profesiones.

En sus discursos, informan de las quejas rutinarias de los clientes relacionadas con la inexactitud del asentamiento cerámico, especialmente en los casos en que los clientes lo desean, colocando la cerámica diagonalmente-45o con la pared. Según los testimonios, en esta región del estado de Paraná, apenas hay un muro o suelo en la plomada y cuadrada, lo que también compromete cualquier asentamiento cerámico.

Acreditan parte de esta responsabilidad a las matemáticas escolares, que según ellos, sólo ha servido para satisfacer las actividades rutinarias de la propia escuela. En sus discursos parece, según la comprensión de Pompeyo y Monteiro (2001) que "vivimos un momento en que la fragmentación del conocimiento limita la comprensión de la realidad, la[porque] organización escolar persiste en mantener un sistema curricular analítico y cartesiano" ( P. 19). Es fundamental, por lo tanto, que la enseñanza de las 
matemáticas escolares contextualice los contenidos, relacionándolos con las actividades desarrolladas por los ciudadanos en los diversos sectores de la actividad humana.

En su argumento crítico, en relación con la forma en que la escuela actualmente enseña matemáticas, el empresario informa que recientemente un Señor - pit-drill, vino varias veces en su tienda pidiendo calcular cuántos metros cúbicos tendría el pozo, que acababa de Taladro. "La última vez que estuviste aquí", dice el empresario, "comentó conmigo que durante 20 años había sido propietario de un aserradero en el estado de Santa Catarina. Le pregunté entonces, "comenta al dueño de la tienda", si sabía cómo Ccabro wood. Para mi sorpresa, el pit puncher respondió rápidamente ":" Seguro, compré y vendí madera, necesitaba saber cuántos metros cúbicos tenía cada tora."

"Cuestioné el ejercicio de pozos sobre lo siguiente"[relata o empresário]: "Si pusiéramos un tronco redondo dent[circular]ro de este pozo, ¿sabrías cómo calcular los metros cúbicos del pozo?" "Sí, hay fácil", respondió el hombre, y según el empresario, en este momento su rostro comenzó a ponerse 'rojo' porque ahora logró hacer la conexión entre las dos situaciones.

Durante nuestra prosa, tanto el empresario como el ingeniero civil acentuaron la falta de interacción entre las matemáticas de la comunidad científica y las matemáticas practicadas en las diversas actividades humanas, debido a que las clases de matemáticas se limitaron a Alcance formal.

Estas situaciones muestran cómo las matemáticas de la escuela se desconectan de las situaciones reales experimentadas por los ciudadanos a diario. Sin embargo, los ciudadanos saben que necesitan este conocimiento, pero tienen dificultades para hacer conexiones. Estas observaciones muestran, en la comprensión de Pompeyo y Monteiro (2001) que "los profesores de hoy tienen un gran reto: educados en un proceso fragmentado, tendrán que superar los límites que esta formación les impone y extrapolar los límites de Contenido visto de manera parcial e histórica "(pág. 15). 
No se trata de sobrevalorar los conocimientos formales o informales. La pregunta que se presenta, busca reflexionar sobre las posibilidades efectivas de relacionar las matemáticas que se enseñan en las escuelas con las prácticas matemáticas practicadas en las actividades humanas. Al hacerlo reflexivamente, buscamos encontrar puntos convergentes, porque según Pompeyo y Monteiro (2001), "si la educación se produce fundamentalmente en una relación" con "el otro, con el mundo, ni el otro ni el mundo pueden verse de manera fragmentada" (pág. 24).

Todas estas perspectivas aclaran lo importante que es en la enseñanza de las matemáticas, el establecimiento de lazos de conocimiento de la comunidad con el conocimiento de la escuela y viceversa. Esta dinámica proporciona una (re) construcción de ambos conocimientos. En teoría, cuando se trabaja en esta perspectiva, la escuela permite a los estudiantes entender mejor y sistemáticamente lo que hacen y por qué proceden matemáticamente.

Según la comprensión de Pompeyo y Monteiro (2001), "un proceso educativo significativo comienza con la interacción de la escuela y la comunidad" (pág. 55), cuyas relaciones pueden resignar el papel de la escuela como un lugar privilegiado para el intercambio de experiencias a Comprender mejor el conocimiento que circula en la vida cotidiana.

La adopción de una práctica pedagógica que deje el tecnicyy y centre la perspectiva de vincular el mundo de la vida con el mundo escolar, puede contribuir a la minimización del proceso de exclusión de un conocimiento matemático crítico reflexivo sobre la realidad. Según D'ambrósio (1996), significa la adopción de una postura socio-interaccionista, buscando un nuevo paradigma educativo que sustituya a la ya desgastada enseñanza-aprendizaje, basada en la relación obsoleta de causa-efecto.

Entendemos y compartimos con Marques (2000) que ningún conocimiento puede ser cerrado en su ámbito de acción. Es necesario socializar, (re) construirse por completo en y por la acción comunicativa establecida entre los sujetos actores. Con y por el uso del lenguaje, desde una perspectiva interactiva-dialogante, se puede proporcionar la 
(re) construcción de ambos aprendizajes, haciendo que las asignaturas sean singularizadas, no sometidas a sujeción.

Que a partir de esta reflexión la práctica pedagógica en el aula puede reorientarse al desarrollo de la plena ciudadanía de los alumnos. Que, según Pinheiro y Rosa (2016), el:

[...] Los profesores de matemáticas se sumergen en la dinámica cultural de los estudiantes y utilizan estrategias de enseñanza y aprendizaje que valoran la dimensión cultural en el aula, para que se pueda desarrollar una educación matemática inclusiva que pueda desarrollarse eficazmente Contribuir a la transformación social (pág. 79).

Una enseñanza con el foco de etnomathematics, creemos para proporcionar a los estudiantes, condiciones para interferir en la realidad a través del conocimiento producido históricamente, colocando al estudiante en el centro del proceso educativo, como una asignatura activa en la (re) construcción de su Conocimiento. Esto se evidenizó en los discursos de los albañiles, porque a partir de su actividad laboral, crean procesos de sus propios pensamientos y estrategias, con inspiración para sus conocimientos previos y aprendizaje adquirido a lo largo de su historia de vida.

\section{OBJETIVOS}

4.1 Contribuir desde la reflexión al educador de matemáticas a adoptar una postura crítica de investigación en relación con la enseñanza de las matemáticas, iniciando su actividad docente a través del establecimiento de vínculos entre matemáticas diarias y matemáticas Escuela 4.2 Contribuir a la Constitución del investigador profesor de su práctica, despertándole a aprender de manera continua para ser profesor, de acuerdo con las necesidades educativas actuales, transformando el aula en un ambiente de intercambio de conocimientos, rescatando Conocimiento histórico socialmente construido por la humanidad; 4.3 Profundizar en la comprensión de la etnomatemática, buscando comprender el papel de los diversos contextos culturales en la educación matemática, gracias a la aproximación entre las matemáticas y la realidad. 


\section{METODOLOGÍA}

Desarrollamos el trabajo a través de diferentes momentos, a saber: estudio teórico sobre el tema Etnomathematics, conversaciones con albañiles y vendedores de tiendas de materiales de construcción. Investigamos dialogadamente con cada profesional sobre los conocimientos matemáticos utilizados en su actividad. Buscamos reflexionar pedagógicamente sobre las posibles conexiones entre las matemáticas y la realidad en la posibilidad de establecer vínculos entre los conocimientos matemáticos utilizados por los albañiles y las matemáticas escolares.

\section{CONTRIBUCION EXPECTADA}

Se espera que la enseñanza de las matemáticas en el aula a través del enfoque etnográfico ${ }^{[9]}$ permite a los profesores y estudiantes aprender sobre la diversidad cultural misma de las matemáticas. Que los resultados de esta investigación ayuden en el desarrollo de una metodología que contribuya a la enseñanza de las matemáticas básicas, con la Etnomatemática como apoyo a esta comprensión.

En palabras de RAM uno,

[...] Enseñar matemáticas en esta concepción permitirá al alumno vincular los conceptos que trabajan en clase con su experiencia diaria, de acuerdo con su entorno natural, social y cultural. No se trata de rechazar las matemáticas académicas, sino de incorporar valores que se experimentan en experiencias grupales, teniendo en cuenta los lazos histórico-culturales (CARNEIRO, 2012, p. 3).

A través del texto producido, el resultado de las lecturas y las sesiones de estudio, se pretende pedagógicamente, la Unión de Teoría y Práctica para la (re) construcción de conocimientos sistematizados. También se espera que las reflexiones resultantes de la lectura del texto permitan una nueva mirada a la realidad y a la educación matemática, desde la perspectiva de la producción de conocimiento desde la práctica diaria y el establecimiento de posibles conexiones entre Matemáticas y realidad. 


\section{REFERENCIAS BIBLIOGRÁFICAS}

BREDA, Adriana LIMA, Valderez Marina do Rosário e GUIMARÃES, Gleny Terezinha Duro. A utilização da Etnomatemática nos cursos de formação continuada de professores: implicações das relações de poder saber na produção de subjetividades. Curitiba. Novembro de 2011. Disponível em: http://educere.bruc.com.br/CD2011/pdf/4668_ 2898.pdf. Acessado em: 20/08/2017.

CARNEIRO, K. T. A. Cultura Surda na aprendizagem matemática da sala de recurso do Instituto Felipe Smaldone: uma abordagem etnomatemática. Anais do 4ํㅡㄹ Congresso Brasileiro de Etnomatemática. Belém, PA: ICEm4, 2012.

D'AMBROSIO, Ubiratan. Etnomatemática - elo entre as tradições e a modernidade. Coleção Tendências em Educação Matemática, 1. Belo Horizonte: Autêntica, 2001, $112 p$.

Etnomatemática: Arte ou técnica de explicar e conhecer. Editora Ática, Série Fundamentos, 2. edição, São Paulo, 1993.

. Educação Matemática: da teoria à prática. Editora Papirus (Coleção Perspectivas em Educação Matemática), Campinas - São Paulo, 1996.

DEMO, Pedro. Pesquisa e construção de conhecimento: Metodologia científica no caminho de Habermas. 3.ed. Rio de Janeiro: Tempo Brasileiro, 1996. 125p.

FIORENTINI, Dario. Alguns modos de ver e conceber o ensino da matemática no Brasil. In. Zetetiké. Campinas, São Paulo, ano 3, n.4,p.1-37, 1995.

GIARDINETTO, José Roberto Boettger. Matemática escolar e matemática da vida cotidiana. Coleção polêmicas do nosso tempo, autores associados, Campinas - São Paulo, 1999, 128p.

LOPES, Antônio José. Os saberes das crianças como ponto de partida para o trabalho pedagógico. In: BRASIL. Secretaria de Educação Básica. Diretoria de Apoio 
à Gestão Educacional. Pacto Nacional pela Alfabetização na Idade Certa: Apresentação / Ministério da Educação, Secretaria de Educação Básica, Diretoria de Apoio à Gestão Educacional. - Brasília: MEC, SEB, 2014.

LINS, R. C.; GIMENEZ, J. Perspectivas em Aritmética e Álgebra para o Século XXI. Série Perspectivas em Educação Matemática. SBEM. Campinas: Papirus Editora, 1997, 176p.

LINS, Rômulo Campos. 0 modelo teórico dos campos semânticos: uma análise epistemológica da álgebra e do pensamento algébrico. In: Dynamis, v.1, n.7, p. 29-39, abr/jun, Blumenau, 1994.

MARQUES, Mario Osorio. A aprendizagem na mediação social do aprendido e da docência. ljuí, Editora UNIJUÍ, 2. edição, 2000, 144 p.

Escrever é Preciso: o princípio da pesquisa. ljuí, Editora UNIJUÍ, Coleção educação, 4. edição, 2001, 163p.

MONTEIRO, A.; POMBEU, G. Jr. A matemática e os temas transversais. Editora Moderna, São Paulo, 2001, 160p.

PINHEIRO, R. C.; ROSA, M. Uma perspectiva etnomatemática para o processo de ensino e aprendizagem de alunos Surdos. RPEM, v. 5, n. 9, p. 56-83, 2016.

ROSA NETO, R. Didática da matemática, São Paulo: Ática, 2002.

ROLIM, Carmem Lucia Artioli. Fórmulas de Silêncio: metodologias no processo de ensino da Matemática. In: SANTOS, Jocyléia Santana. ZAMBONI, Ernesta. Potencialidades Investigativas da Educação. Goiânia: Ed. da PUC Goiás, 2010.

SANTOS, Lijecson Souza dos. Contextualização Matemática Em Situação De Ensino E Aprendizagem No Eja. Disponível em: https://editorarealize.com.br/revistas/cintedi/trabalhos/TRABALHO_EV060_MD1_SA 18_ID 492_23102016194610.pdf. 2016. Acesso em 11/11/2017, Hora: 22hrs e 38 min. 
SILVA. Adelmo Carvalho da. et. al. Paradigmas Educacionais: Contribuições para o Ensino de Matemática. In. SILVA, Adelmo Carvalho. et al, Ensinar matemática: Formação, investigação e práticas docentes. Cuiabá, MT: DUFMT, 2012.

SKOVSMOSE, Ole. Um convite à educação matemática crítica. Tradução de Orlando de Andrade Figueiredo. Campinas: Papirus, 2014.

VYGOTSKY, Lev Seminovich. A construção do pensamento e da linguagem. Tradução: Paulo Bezerra, São Paulo, Martins Fontes, 2001, 496p.

8. El conocimiento, en esta perspectiva se entiende como una afirmación de creencias, junto con una justificación para la afirmación de creencias.

9. En este trabajo se utilizó para designar la investigación de las concepciones, tradiciones y prácticas matemáticas de un grupo[pedreiros] social y el trabajo pedagógico que se puede desarrollar en la perspectiva que el grupo interpreta y codifica sus conocimientos; Adquirir los conocimientos producidos por las matemáticas académicas, utilizando, cuando se enfrenta a situaciones contextualizadas, la que parece más apropiada.

Enviado: Junio de 2019.

Aprobado: Julio de 2019. 